\title{
Probiotics Improve Chemerin Levels and Metabolic Syndrome Parameters in Obese Rats
}

\author{
Menşure Nur Çelik1, Mehtap Ünlü Söğüt2 \\ ${ }^{1}$ Department of Nutrition and Dietetics, Gazi University School of Health Sciences, Ankara, Turkey \\ ${ }^{2}$ Department of Nutrition and Dietetics, Ondokuz Mayıs University School of Health Sciences, Samsun, Turkey
}

Background: Chemerin is a recently discovered adipokine that plays a role in adipocyte metabolism. It is a novel chemoattractant adipokine whose expression and secretion are increased by adipogenesis.

Aims: To evaluate the effects of probiotic supplementation on chemerin level, inflammation, and metabolic syndrome components in obese Wistar rats.

Study Design: Animal experiment.

Methods: We divided the experimental animals into three groups, each consisting of eight rats. Group 1 was the control group. Group 2 was the experimentally obese group, in which rats were fed with a high-fat diet. Group 3 was the obese intervention group, in which rats were supplemented with probiotics after obesity induction.

Results: At the end of the study, a statistically significant difference was found between the groups in final weights, weight changes, and body mass index values $(\mathrm{p}<0.05)$. Weight gain was $34.12 \pm 3.70 \mathrm{~g}$ in group 3 post-probiotic supplementation and $53.25 \pm 8.35 \mathrm{~g}$ in group $2(\mathrm{p}<0.05)$. Obese rats showed increased levels of fasting plasma glucose, insulin, insulin resistance (homeostatic model assessmentinsulin resistance), total cholesterol, low-density lipoprotein cholesterol, inflammatory markers, and leptin compared to those in the control group. Chemerin levels were $14.31 \pm 13.34 \mathrm{ng} / \mathrm{mL}$ in group 2 and $2.67 \pm 2.42 \mathrm{ng} / \mathrm{mL}$ in group $3(\mathrm{p}<0.05)$.

Conclusion: Probiotic supplementation (group 3 ) reduced weight gain, and there were positive effects on the levels of fasting plasma glucose, insulin, homeostatic model assessment-insulin resistance, triglycerides, inflammatory markers, leptin, and chemerin.

Keywords: Chemerine protein, metabolic syndrome, obesity, probiotics, rat
The World Health Organization defines obesity as abnormal or excessive fat accumulation caused by an imbalance between energy intake and energy expenditure (1). Worldwide, 390 million adult women and 281 million adult men were reported to be obese in 2016 (2). In recent years, intestinal microflora emerged as important components for the prevention of obesity and related diseases as well as for supporting treatment. Regulation of intestinal microflora (such as by antibiotic, prebiotic, and probiotic use), and the effectiveness of treatment for obesity and related diseases are being investigated using human studies following successful animal studies (3-5). Probiotics, which are viable microorganisms with beneficial health effects, when taken in sufficient quantities have the potential to reduce obesity by decreasing fat storage and increasing satiety and energy expenditure (6).

The strong association between diet, intestinal microbiota, and obesity has been an important research topic in recent years to better understand the etiology of obesity and to develop new treatment methods (7). Especially, recent metagenomics-based studies have shown that intestinal microbiota affects not only the energy balance but also the immune and intestinal barrier functions and acts as a factor affecting the entire body metabolism $(8,9)$.

Chemerin is a recently discovered adipokine that has been demonstrated to play a role in adipogenesis and adipocyte metabolism $(10,11)$. Chemerin expression and secretion are known to be increased by adipogenesis (12). Therefore, it is important to determine and regulate circulating levels of chemerin as they are associated with an increased risk of developing obesity, type 2 diabetes mellitus, cardiovascular diseases, inflammation, metabolic syndrome, and several other diseases $(13,14)$.

Studies investigating the relationship between chemerin and obesity have reported that individuals who followed various methods for weight loss have low serum chemerin levels compared to those in obese individuals without weight loss, and the decrease in the serum concentrations of chemerin was found to be associated with the improvement in weight loss and metabolic parameters (15-18).

Metabolic syndrome is a combination of several cardiovascular risk factors that are closely associated with abdominal adiposity and insulin resistance (18). Circulating chemerin levels are known to

Address for Correspondence: Menșure Nur Çelik, Department of Nutrition and Dietetics, Gazi University School of Health Sciences, Ankara, Turkey Phone: +90 $3122162969 \quad$ e-mail: dyt.mensurenurcelik@gmail.com ORCID: orcid.org/0000-0002-6524-7114

Received: 15 February 2019 Accepted: 1 July 2019•DOI: 10.4274/balkanmedj.galenos.2019.2019.2.61

Available at www.balkanmedicaljournal.org

Cite this article as:

Çelik MN, Ünlü Söğüt M. Probiotics Improve Chemerin Levels and Metabolic Syndrome Parameters in Obese Rats. Balkan Med J 2019;36:270-5

${ }^{\circ}$ Copyright 2019 by Trakya University Faculty of Medicine / The Balkan Medical Journal published by Galenos Publishing House. 
increase in individuals with obesity and positively correlate with metabolic syndrome markers, including body mass index, waist/ hip ratio, systolic blood pressure, and serum triglyceride levels (19).

In this study, we evaluated the effects of probiotics on diabetes, insulin resistance, lipid profile, obesity, inflammation, and chemerin levels in obese rats.

\section{MATERIALS AND METHODS}

\section{Experimental animals}

The Ondokuz Mayis University Experimental Animal Application and Research Center supplied 24 male rats aged 4-6 weeks with an average weight of 270-290 g. All rats were housed under standard conditions (such as heat, humidity, light, and ventilation) and fed ad libitum with dry chow (20-25 g/day per rat) during the study. All rats were housed in plastic cages at $24{ }^{\circ} \mathrm{C} \pm 3{ }^{\circ} \mathrm{C}$ in $12-\mathrm{h}$ light/12-h dark cycles with 40\%-60\% humidity in the laboratories of the Ondokuz Mayıs University. Rats had free access to water and food. Ethical approval was obtained from the Ondokuz May1s University Ethical Committee of Animal Experiments (IRB number: 28.12.2016/12).

\section{Experimental design and animal grouping}

The experiment lasted 16 weeks (w) and was divided into the following two phases: 1 ) induction of obesity [diet-induced obesity (DIO)] (0-8 w) and 2) intervention (9-16 w). The 24 rats were randomized into three groups containing eight rats in each group. Group 1 was the control group fed with a standard diet, group 2 was the study group fed with a high-fat diet (HFD) (60\% fat), and group 3 was another study group fed with HFD supplemented with probiotics (Figure 1).

During the entire duration of the experiment, body weights of the rats were recorded weekly and mean body weight and weight gain were calculated. For the evaluation of obesity, body mass index [body weight $(\mathrm{g}) /$ height $\left(\mathrm{cm}^{2}\right)$ ], which was calculated by measuring the body weight and the naso-anal length, was used. Rats with a body mass index $>0.68 \mathrm{~g} / \mathrm{cm}^{2}$ were considered as obese (20).

\section{DIO rats and diets of animals}

Rats in group 1 were fed with standard rat chow (16\% carbohydrate, $54 \%$ protein, and $30 \%$ fat) throughout the study (16 w). Rats in group 2 and group 3 were fed with HFD (8.9\% carbohydrate, $30.8 \%$ protein, and $60.3 \%$ fat) for $8 \mathrm{w}$ to stimulate DIO. After $8 \mathrm{w}$, DIO rats in group 2 continued eating the HFD. However, the DIO

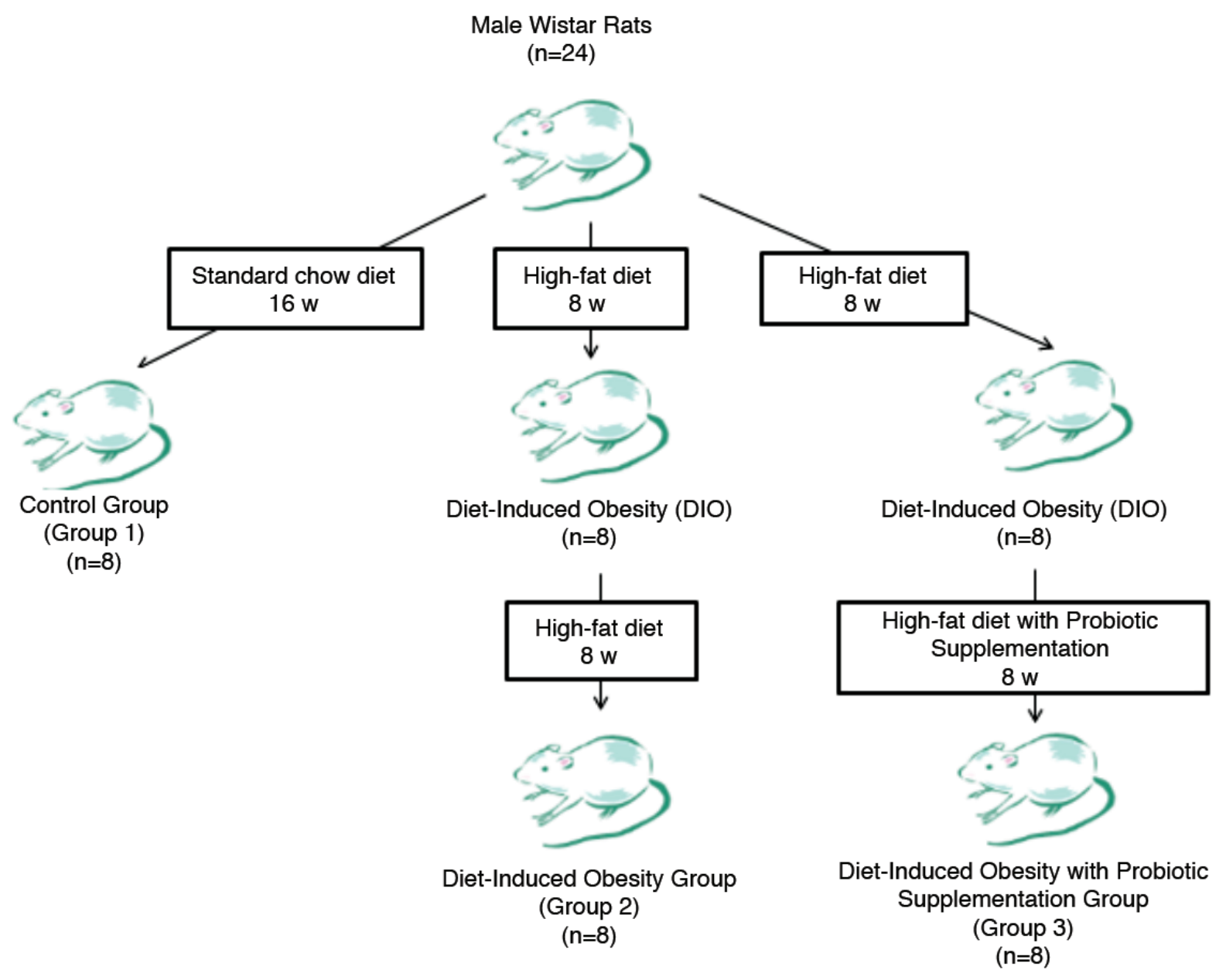

FIG. 1. Flow diagram outlining the experimental design. 
rats in group 3 received probiotic supplementation via oral gavage in addition to the HFD.

\section{Probiotic administration protocol}

A pool of probiotics that included Lactobacillus acidophilus, Bacillus lactis, Lactobacillus paracasei, and Lactobacillus rhamnosus (Solgar ${ }^{\circledR}$, Advanced Multi-Billion Dophilus ${ }^{\mathrm{TM}}$, Turkey) was given for $8 \mathrm{w}$ daily $\left(6 \times 10^{8}\right.$ of each strain; final concentration $2.4 \times 10^{9}$ cfu bacteria). Prior to gavage, the probiotics were diluted in sterile water $(21)$.

\section{Blood samples}

The animals were decapitated under anesthesia, and the maximum amount of blood that could be collected directly was used for biochemical analysis. Blood samples were collected in anticoagulant-free biochemical tubes, centrifuged at $3000 \mathrm{rpm}$ for $10 \mathrm{~min}$, and the resulting sera were stored at $-80{ }^{\circ} \mathrm{C}$ until analysis.

\section{Biochemical analyses of serum}

Insulin and fasting plasma glucose levels were analyzed as an indicator of diabetes; the levels of the cytokines interleukin-6 (IL-6), IL-10, and tumor necrosis factor- $\alpha$ and C-reactive protein were analyzed as indicators of inflammation; leptin level was evaluated as an indicator of obesity; triglyceride, total cholesterol, high-density lipoprotein cholesterol, and low-density lipoprotein cholesterol levels were determined to evaluate the lipid profile; and serum levels of chemerin were analyzed in serum samples. For this analysis, the enzyme-linked immunosorbent assay technique was applied using a commercially available kit (Rel Assay Diagnostics $^{\circledR}$, Turkey).

Insulin resistance was measured using the homeostasis model assessment of insulin resistance $=$ fasting insulin $(\mu \mathrm{U} / \mathrm{mL}) \times$ fasting glucose $(\mathrm{mmol} / \mathrm{L}) / 22.5$. An indirect measure of insulin sensitivity was calculated using the quantitative insulin sensitivity check index as follows: $1 /[\log ($ fasting insulin $\mu \mathrm{U} / \mathrm{mL})+\log ($ fasting glucose $\mathrm{mg} / \mathrm{dL})]$.

\section{Statistical analysis}

Data were tested for normal distribution using the Shapiro-Wilk test. When the criteria for normal distribution were not achieved, the nonparametric Kruskal-Wallis and Mann-Whitney U pairwise comparison tests were used. The Mann-Whitney U test was performed to assess the significance of pairwise differences using Bonferroni correction to adjust for multiple comparisons. An overall 5\% type-I error level was used to determine the statistical significance. Results are presented as mean \pm standard deviations. Values were considered to be significant when $\mathrm{p}<0.05$; different superscript letters $(a, b)$ were used to indicate significant variations at $\mathrm{p}<0.05$ in the tables. Considering leptin values, the minimum number of samples for $95 \%$ confidence, a significant difference of 0.55 , a standard deviation of 0.35 , and a test power of 0.80 was eight rats (per group) (22,23). Statistical analyses were performed using the SPSS (Statistical Package for the Social Sciences) 21 application. A minimum difference of 0.55 , a standard deviation of 0.35 , and a test power of 0.80 , for $95 \%$ confidence, taking into account the minimum number of samples, the leptin value, were eight rats.

\section{RESULTS}

\section{Evaluation of morphometric measurements}

DIO rats were fed with HFD with or without probiotic supplementation for $16 \mathrm{w}$. Table 1 shows the initial and final weights, body mass index values, and weight changes of the rats. At baseline, there was no significant difference in body weight between the groups $(\mathrm{p}>0.05)$. After the first $8 \mathrm{w}$, the average weight gain was $63.37 \pm 9.69 \mathrm{~g}$ in group 2 and $90.50 \pm 29.07 \mathrm{~g}$ in group 3. Between weeks 8 and 16, the average weight gain was $53.25 \pm 23.62 \mathrm{~g}$ in group 2 and $34.12 \pm 10.46 \mathrm{~g}$ in group 3 . Weight changes and body mass index values were significantly increased at 8 and $16 \mathrm{w}$ in rats fed with HFD $(\mathrm{p}<0.05)$. The changes in weight and weight gain recorded during the study are shown in Figure 2. However, despite having a higher body mass index than the other groups at week 8 , group 3 showed a significant decrease in the rate of body mass index increase after receiving probiotics. Figure 3 shows the body mass index changes observed during the study.

\section{Evaluation of biochemical parameters}

To evaluate the effects of HFD and probiotic supplementation on biochemical parameters in DIO rats, we examined the levels of fasting plasma glucose, insulin, inflammatory markers, chemerin, and leptin and the lipid profile. Table 2 shows the biochemical parameters of the rats.

Insulin levels, insulin resistance measured using homeostasis model assessment of insulin resistance, and insulin sensitivity measured using quantitative insulin sensitivity check index were significantly decreased in rats after probiotic supplementation compared to those in rats fed with HFD without supplementation. Chemerin levels were significantly increased in response to HFD (group 2) but significantly recovered after probiotic supplementation (group 3) (Figure 4). No statistically significant difference was observed in the remaining parameters $(\mathrm{p}>0.05)$.

TABLE 1. Effects of normal diet and HFD on body weight and BMI in rats

\begin{tabular}{|c|c|c|c|c|}
\hline & Group $1(\mathrm{n}=8)$ & Group $2(\mathrm{n}=8)$ & Group $3(n=8)$ & $\mathrm{p}$ \\
\hline \multicolumn{5}{|c|}{ Body weight (g) } \\
\hline Baseline & $275.25 \pm 11.02$ & $294.63 \pm 10.15$ & $292.25 \pm 18.31$ & 0.650 \\
\hline 8 week & $288.63 \pm 7.18$ & $358.00 \pm 18.47^{a}$ & $382.75 \pm 11.11^{b}$ & $0.000 *$ \\
\hline $\begin{array}{l}\text { Final (16 } \\
\text { week) }\end{array}$ & $336.13 \pm 10.02$ & $411.25 \pm 29.6^{\mathrm{a}}$ & $416.88 \pm 18.94$ & $0.000 *$ \\
\hline $\begin{array}{l}\text { Weight gain } \\
(0-8 \text { week })\end{array}$ & $53.37 \pm 10.75$ & $63.37 \pm 9.69$ & $90.50 \pm 29.07$ & $0.021 *$ \\
\hline $\begin{array}{l}\text { Weight gain } \\
\text { (8-16 week) }\end{array}$ & $47.50 \pm 6.80$ & $53.25 \pm 23.62$ & $34.12 \pm 10.46$ & 0.320 \\
\hline \multicolumn{5}{|l|}{ BMI (g/cm²) } \\
\hline Baseline & $0.44 \pm 0.02$ & $0.55 \pm 0.01^{\mathrm{a}}$ & $0.55 \pm 0.34$ & $0.000 *$ \\
\hline 8 week & $0.54 \pm 0.01$ & $0.68 \pm 0.03^{a}$ & $0.72 \pm 0.02^{b}$ & $0.000 *$ \\
\hline $\begin{array}{l}\text { Final (16 } \\
\text { week) }\end{array}$ & $0.63 \pm 0.01$ & $0.78 \pm 0.56^{\mathrm{a}}$ & $0.78 \pm 0.03$ & $0.000^{*}$ \\
\hline \multicolumn{5}{|c|}{$\begin{array}{l}{ }^{\mathrm{a}} \mathrm{p}<0.05 \text { (compared with group } 1 \text { ); }{ }^{\mathrm{b}} \mathrm{p}<0.05 \text { (compared with group } 2 \text { ); }{ }^{*} \mathrm{p}<0.05 \text {; BMI: } \\
\text { body mass index; Group } 1 \text { : control group; Group } 2 \text { : diet-induced obesity group; Group } \\
\text { 3: diet-induced obesity with probiotic supplementation group; HFD: high-fat diet }\end{array}$} \\
\hline
\end{tabular}


TABLE 2. Effects of HFD and probiotic supplementation on biochemical parameters in DIO rats

\begin{tabular}{lccc}
\hline & Group 1 $(\mathrm{n}=8)$ & Group 2 $(\mathrm{n}=8)$ & Group 3 $(\mathrm{n}=8)$ \\
\hline FPG $(\mathrm{mg} / \mathrm{dL})$ & $194.32 \pm 10.99$ & $212.13 \pm 45.11$ & $199.23 \pm 18.64$ \\
Insulin (mIU/L) & $25.28 \pm 10.26$ & $27.97 \pm 10.61$ & $14.44 \pm 1.30^{\mathrm{b}}$ \\
HOMA-IR & $11.06 \pm 4.29$ & $14.55 \pm 6.36$ & $7.30 \pm 0.89^{\mathrm{b}}$ \\
QUICKI & $0.27 \pm 0.01$ & $0.26 \pm 0.01$ & $0.28 \pm 00^{\mathrm{b}}$ \\
TC $(\mathrm{mmol} / \mathrm{L})$ & $2.64 \pm 0.71$ & $4.60 \pm 1.13$ & $4.69 \pm 0.64$ \\
TG $(\mathrm{mmol} / \mathrm{L})$ & $2.20 \pm 0.57$ & $1.81 \pm 0.80$ & $1.50 \pm 0.26$ \\
HDL-C $(\mathrm{ng} / \mathrm{mL})$ & $71.40 \pm 33.46$ & $74.59 \pm 39.72$ & $48.43 \pm 10.89$ \\
LDL-C $(\mu \mathrm{g} / \mathrm{mL})$ & $6.25 \pm 0.28$ & $7.12 \pm 0.70$ & $6.91 \pm 0.17$ \\
IL-6 $(\mathrm{pg} / \mathrm{mL})$ & $215.41 \pm 43.66$ & $215.62 \pm 27.27$ & $205.00 \pm 26.33$ \\
IL-10 $(\mathrm{pg} / \mathrm{mL})$ & $388.93 \pm 151.17$ & $286.56 \pm 120.87$ & $348.36 \pm 136.80$ \\
CRP $(\mathrm{mg} / \mathrm{L})$ & $4.04 \pm 1.73$ & $4.15 \pm 1.12$ & $4.55 \pm 1.10$ \\
TNF- $\alpha(\mathrm{ng} / \mathrm{L})$ & $283.22 \pm 196.08$ & $485.77 \pm 271.99$ & $336.52 \pm 142.42$ \\
Leptin $(\mathrm{ng} / \mathrm{L})$ & $434.34 \pm 167.81$ & $311.79 \pm 123.62$ & $331.72 \pm 24.16$ \\
Chemerin $(\mathrm{ng} / \mathrm{mL})$ & $0.78 \pm 0.39$ & $14.31 \pm 13.3^{\mathrm{a}}$ & $2.67 \pm 2.43^{\mathrm{b}}$ \\
\hline
\end{tabular}

${ }^{a} p<0.05$ (compared with group 1); $b_{p}<0.05$ (compared with group 2); CRP: C-reactive protein; DIO: diet-induced obesity; FPG: fasting plasma glucose; HDL-C: high-density lipoprotein cholesterol; HFD: high-fat diet; HOMA-IR: homeostasis model assessment of insulin resistance; IL: interleukin; LDL-C: low-density lipoprotein cholesterol; QUICKI: quantitative insulin sensitivity check index; TC: total cholesterol; TG: triglyceride; TNF- $\alpha$ : tumor necrosis factor $\alpha$

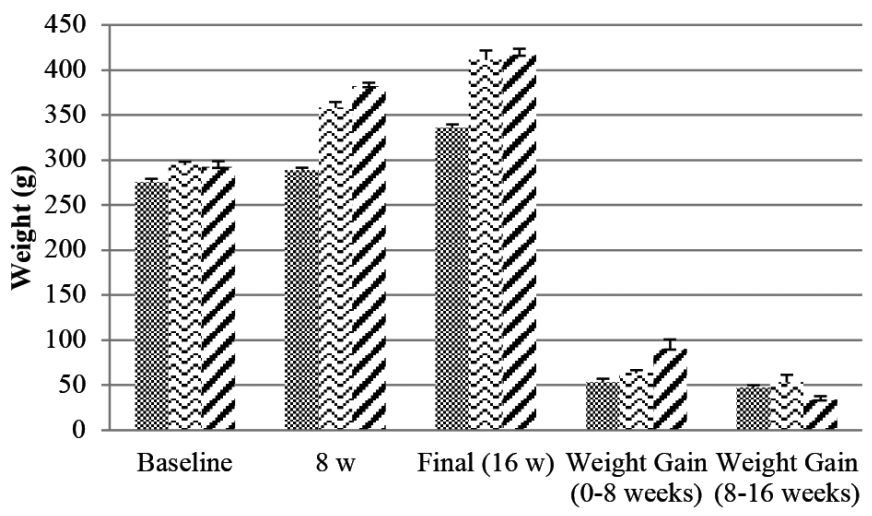

* Group $1 \quad \therefore$ Group $2 \quad$-Group 3

FIG. 2. At baseline, there was no difference in body weight between groups. Body weight changes and weight gains at $8 \mathrm{w}$ and $16 \mathrm{w}$ are shown.

Group 1: control; Group 2: diet-induced obesity; Group 3: diet-induced obesity with probiotic supplementation

\section{DISCUSSION}

In recent years, it has been suggested that regulation of microbiota by various mechanisms using probiotics plays a role in preventing obesity and related diseases (6).

It has been reported that diets in which $45 \%-60 \%$ of the energy is derived from fats provide $20 \%-40 \%$ of weight gain $(24,25)$. Our results were similar to the literature, and the mean body weight and weight gain (weight increase of $21 \%$ at $8 \mathrm{w}$ and $39 \%$ at $16 \mathrm{w}$ ), due to increased adipogenesis, in DIO rats were higher than those in the control group $(\mathrm{p}<0.05)$. Furthermore, rats in group 3 were appeared

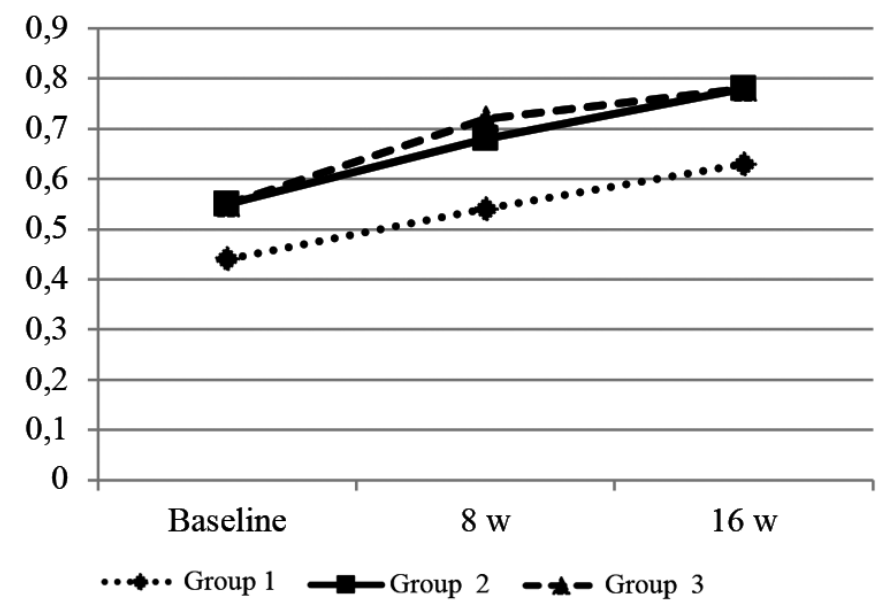

FIG. 3. The figure shows that BMI changes between groups. BMI values were rapidly increased in group 2. However, BMI values tended to increase slowly after the start of probiotic supplementation in group 3 .

Group 1: control; Group 2: diet-induced obesity; Group 3: diet-induced obesity with probiotic supplementation

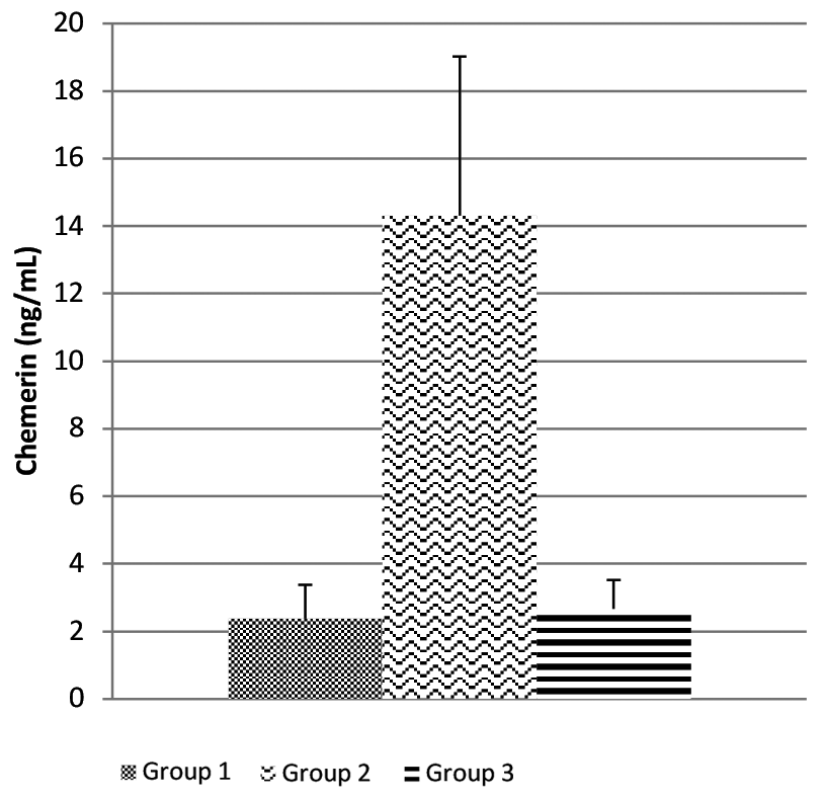

FIG. 4. The figure shows that mean levels of chemerin adipokine according to groups. Chemerin significantly increased in response to a HFD (group 2), but significantly recovered after introduction of probiotic supplementation (group 3).

Group 1: control; Group 2: diet-induced obesity; Group 3: diet-induced obesity with probiotic supplementation

to have more weight gain than rats in group 2, but the difference was not significant.

Probiotics can affect metabolic syndrome, type 2 diabetes mellitus, and obesity by regulating intestinal microbiota, favoring insulin signaling, and decreasing cholesterol levels. Evidence obtained from animal studies indicates that probiotic supplementation partially reduces weight gain and positively affects parameters related to obesity and HFD-related diseases $(26,27)$. In our study, body weight gain was decreased in DIO rats that received probiotic 
supplementation (group 3) compared to that in DIO rats (group 2). After probiotic supplementation in our study, the levels of fasting plasma glucose, insulin, and homeostasis model assessment of insulin resistance were found to be consistent with the literature. HFD causes adverse changes in the lipid profile, which can be normalized after probiotic supplementation $(28,29)$. Some studies observed no effect after probiotic supplementation on total cholesterol, high-density lipoprotein cholesterol, lowdensity lipoprotein cholesterol, and triglyceride levels $(30,31)$. Results of our study also demonstrated that although there was no significant difference in total cholesterol, low-density lipoprotein cholesterol, and high-density lipoprotein cholesterol levels after probiotic supplementation, the decrease in triglyceride levels was similar to the variable results of the lipid profile reported in the literature.

Research shows that supplementation with probiotics to HFD reduces leptin levels compared with standard diets (32). In a similar study, no difference was observed in leptin levels between groups after 12 weeks of probiotic supplementation (33). In our study, we found that leptin levels were decreased in DIO rats after probiotic supplementation (group 3) compared to those in DIO rats (group 2 ), although the difference was not significant.

Supplementation of probiotics to rats fed with HFD resulted in an anti-inflammatory response demonstrated by the reduction in the levels of proinflammatory cytokines such as IL-6, IL-17, and tumor necrosis factor- $\alpha$. Results obtained from animal experiments suggest that supplementation of probiotics to HFD stimulates an anti-inflammatory response $(29,32)$. Another study reported that probiotic supplementation to HFD did not alter tumor necrosis factor- $\alpha$ and IL-6 levels (33). In our study, the levels of IL-6, IL10 , tumor necrosis factor- $\alpha$, and C-reactive protein were decreased in DIO rats that received probiotic supplementation (group 3) compared to those in DIO rats (group 2), although the difference did not reach statistical significance.

It is known that adipogenesis increases the expression and secretion of the adipokine chemerin $(10,12)$. Studies have shown that rats fed with HFD gained more weight and had higher levels of chemerin than those fed with a standard diet $(34,35)$. Similarly, the weight increase in rats fed with HFD was associated with an increase in chemerin levels in our study. Rats fed with HFD showed higher levels of chemerin than the control group $(p<0.05)$.

Studies have shown that chemerin levels are associated with metabolic syndrome markers $(17,36)$. In our study, increased levels of chemerin were found to be associated with elevated levels of fasting plasma glucose, insulin, homeostasis model assessment of insulin resistance, total cholesterol, high-density lipoprotein cholesterol, and low-density lipoprotein cholesterol and reduced levels of triglyceride in DIO rats. Chemerin levels were decreased in DIO rats after probiotic supplementation $(\mathrm{p}<0.05)$.

In conclusion, although this study demonstrates a relationship between chemerin levels and metabolic syndrome components, the effect of probiotic supplementation on serum levels of chemerin, and hence obesity and related diseases, is unknown. No studies have yet investigated the effect of probiotics on chemerin levels, although probiotics are known to be related to diseases and are a current topic under investigation. Therefore, our study contributes to the literature as the first investigation to evaluate these parameters together.

For this purpose, it is important to show that chemerin is a new modifiable factor of obesity affected by probiotic supplementation. Results of this study obtained from obese rats would provide both weight control and reduction risk of developing obesity-related diseases by increasing the levels of chemerin to the desired level with probiotic supplementation in the diet of obese individuals.

Conflict of Interest: No conflict of interest was declared by the authors.

Financial Disclosure: This work was financially supported by Ondokuz Mayis University Scientific Research Projects Commission (PYO.SBF.1904.17.008).

\section{REFERENCES}

1. NCD Risk Factor Collaboration (NCD-RisC). Trends in adult body-mass index in 200 countries from 1975 to 2014: a pooled analysis of 1698 population-based measurement studies with 19·2 million participants. Lancet 2016;387:1377-96.

2. NCD Risk Factor Collaboration (NCD-RisC). Worldwide trends in body-mass index, underweight, overweight, and obesity from 1975 to 2016: a pooled analysis of 2416 population-based measurement studies in 128.9 million children, adolescents, and adults. Lancet 2017;390:2627-42.

3. Carvalho BM, Guadagnini D, Tsukumo DML, Schenka AA, Latuf-Filho P, Vassallo J, et al. Modulation of gut microbiota by antibiotics improves insulin signalling in highfat fed mice. Diabetologia 2012;55:2823-34.

4. Gomes AC, Bueno AA, de Souza RG, Mota JF. Gut microbiota, probiotics and diabetes. Nutr J 2014;13:60.

5. Kootte RS, Vrieze A, Holleman F, Dallinga-Thie GM, Zoetendal EG, de Vos WM, et al. The therapeutic potential of manipulating gut microbiota in obesity and type 2 diabetes mellitus. Diabetes Obes Metab 2012;14:112-20.

6. Sanchez M, Darimont C, Panahi S, Drapeau V, Marette A, Taylor VH, et al. Effects of a Diet-Based Weight-Reducing Program with Probiotic Supplementation on Satiety Efficiency, Eating Behaviour Traits, and Psychosocial Behaviours in Obese Individuals. Nutrients 2017;9. pii: E284.

7. Sonnenburg JL, Bäckhed F. Diet-microbiota interactions as moderators of human metabolism. Nature 2016;535:56-64.

8. Bäckhed F, Ding H, Wang T, Hooper LV, Koh GY, Nagy A, et al. The gut microbiota as an environmental factor that regulates fat storage. Proc Natl Acad Sci 2004;101:15718-23.

9. Turnbaugh PJ, Ley RE, Mahowald MA, Magrini V, Mardis ER, Gordon JI. An obesity-associated gut microbiome with increased capacity for energy harvest. Nature 2006;444:1027-31.

10. Goralski KB, McCarthy TC, Hanniman EA, Zabel BA, Butcher EC, Parlee SD, et al Chemerin, a novel adipokine that regulates adipogenesis and adipocyte metabolism. $\mathrm{J}$ Biol Chem 2007;282:28175-88.

11. Piya MK, McTernan PG, Kumar S. Adipokine inflammation and insulin resistance: the role of glucose, lipids and endotoxin. J Endocrinol 2013;216:T1-15.

12. Chang SS, Eisenberg D, Zhao L, Adams C, Leib R, Morser J, et al. Chemerin activation in human obesity. Obesity (Silver Spring) 2016;24:1522-9.

13. Ernst MC, Issa M, Goralski KB, Sinal CJ. Chemerin exacerbates glucose intolerance in mouse models of obesity and diabetes. Endocrinology 2010;151:1998-2007.

14. Ernst MC, Sinal CJ. Chemerin: at the crossroads of inflammation and obesity. Trends Endocrinol Metab 2010;21:660-7.

15. Bozaoglu K, Bolton K, McMillan J, Zimmet P, Jowett J, Collier G, et al. Chemerin is a novel adipokine associated with obesity and metabolic syndrome. Endocrinology 2007;148:4687-94.

16. Fatima SS, Bozaoglu K, Rehman R, Alam F, Memon AS. Elevated chemerin levels in Pakistani men: an interrelation with metabolic syndrome phenotypes. PLoS One 2013;8:e57113.

17. Sell H, Divoux A, Poitou C, Basdevant A, Bouillot JL, Bedossa P, et al. Chemerin correlates with markers for fatty liver in morbidly obese patients and strongly decreases after weight loss induced by bariatric surgery. J Clin Endocrinol Metab 2010;95:2892-6. 
18. Li Y, Shi B, Li S. Association between serum chemerin concentrations and clinical indices in obesity or metabolic syndrome: a meta-analysis. PLoS One 2014;9:e113915

19. Wassink AM, Olijhoek JK, Visseren FL. The metabolic syndrome: metabolic changes with vascular consequences. Eur J Clin Invest 2007;37:8-17.

20. Novelli EL, Diniz YS, Galhardi CM, Ebaid GM, Rodrigues HG, Mani F, et al. Anthropometrical parameters and markers of obesity in rats. Lab Anim 2007;41:111-9.

21. Bagarolli RA, Tobar N, Oliveira AG, Araújo TG, Carvalho BM, Rocha GZ, et al Probiotics modulate gut microbiota and improve insulin sensitivity in DIO mice. J Nutr Biochem 2017;50:16-25.

22. Desmarchelier C, Ludwig T, Scheundel R, Rink N, Bader BL, Klingenspor M, et al. Diet-induced obesity in ad libitum-fed mice: food texture overrides the effect of macronutrient composition. Br J Nutr 2013;109:1518-27.

23. Cohen J. Statistical power analysis for the behavioral sciences: Routledge; 2013.

24. Lamont BJ, Waters MF, Andrikopoulos S. A low-carbohydrate high-fat diet increases weight gain and does not improve glucose tolerance, insulin secretion or $\beta$-cell mass in NZO mice. Nutr Diabetes 2016;6:e194.

25. Qin L, Zhao Y, Zhang B, Li Y. Amentoflavone improves cardiovascular dysfunction and metabolic abnormalities in high fructose and fat diet-fed rats. Food Funct 2018;9:243-52.

26. Lecomte V, Kaakoush NO, Maloney CA, Raipuria M, Huinao KD, Mitchell HM, et al Changes in gut microbiota in rats fed a high fat diet correlate with obesity-associated metabolic parameters. PLoS One 2015;10:e0126931.

27. Nunez IN, Galdeano CM, de LeBlanc Ade M, Perdigon G. Evaluation of immune response, microbiota, and blood markers after probiotic bacteria administration in obese mice induced by a high-fat diet. Nutrition 2014;30:1423-32.
28. Mihailović M, Živković M, Jovanović J, Tolinački M, Sinadinović M, Rajić J, et al. Oral administration of probiotic Lactobacillus paraplantarum BGCG11 attenuates diabetes-induced liver and kidney damage in rats. J Funct Foods 2017;38:427-37.

29. Shin JH, Nam MH, Lee H, Lee JS, Kim H, Chung MJ, et al. Amelioration of obesityrelated characteristics by a probiotic formulation in a high-fat diet-induced obese rat model. Eur J Nutr 2018;57:2081-90.

30. Kang JH, Yun SI, Park HO. Effects of Lactobacillus gasseri BNR17 on body weight and adipose tissue mass in diet-induced overweight rats. J Microbiol 2010;48:712-4.

31. Shirouchi B, Nagao K, Umegatani M, Shiraishi A, Morita Y, Kai S, et al. Probiotic Lactobacillus gasseri SBT2055 improves glucose tolerance and reduces body weight gain in rats by stimulating energy expenditure. Br J Nutr 2016;116:451-8.

32. Chang CJ, Lu CC, Lin CS, Martel J, Ko YF, Ojcius DM, et al. Antrodia cinnamomea reduces obesity and modulates the gut microbiota in high-fat diet-fed mice. Int J Obes (Lond) 2018;42:231-43.

33. Karimi G, Jamaluddin R, Mohtarrudin N, Ahmad Z, Khazaai H, Parvaneh M. Singlespecies versus dual-species probiotic supplementation as an emerging therapeutic strategy for obesity. Nutr Metab Cardiovasc Dis 2017;27:910-8.

34. Roh SG, Song SH, Choi KC, Katoh K, Wittamer V, Parmentier M, et al. Chemerin a new adipokine that modulates adipogenesis via its own receptor. Biochem Biophys Res Commun 2007;362:1013-8.

35. Lloyd JW, Zerfass KM, Heckstall EM, Evans KA. Diet-induced increases in chemerin are attenuated by exercise and mediate the effect of diet on insulin and HOMA-IR. Ther Adv Endocrinol Metab 2015;6:189-98.

36. Ress C, Tschoner A, Engl J, Klaus A, Tilg H, Ebenbichler CF, et al. Effect of bariatric surgery on circulating chemerin levels. Eur J Clin Invest 2010;40:277-80. 